\title{
On the origin of asymmetries in bilateral supernova remnants
}

\author{
S. Orlando ${ }^{1,2}$, F. Bocchino ${ }^{1,2}$, F. Reale ${ }^{3,1,2}$, G. Peres ${ }^{3,1,2}$, and O. Petruk ${ }^{4,5}$ \\ 1 INAF - Osservatorio Astronomico di Palermo "G.S. Vaiana", Piazza del Parlamento 1, 90134 Palermo, Italy \\ e-mail: orlando@astropa.inaf.it \\ 2 Consorzio COMETA, via Santa Sofia 64, 95123 Catania, Italy \\ 3 Dip. di Scienze Fisiche \& Astronomiche, Univ. di Palermo, Piazza del Parlamento 1, 90134 Palermo, Italy \\ 4 Institute for Applied Problems in Mechanics and Mathematics, Naukova St. 3-b Lviv 79060, Ukraine \\ 5 Astronomical Observatory, National University, Kyryla and Methodia St. 8 Lviv 79008, Ukraine
}

Received 14 July 2006 / Accepted 30 March 2007

ABSTRACT

\begin{abstract}
Aims. We investigate whether the morphology of bilateral supernova remnants (BSNRs) observed in the radio band is determined mainly either by a non-uniform interstellar medium (ISM) or by a non-uniform ambient magnetic field.

Methods. We perform 3D MHD simulations of a spherical SNR shock propagating through a magnetized ISM. Two cases of shock propagation are considered: 1) through a gradient of ambient density with a uniform ambient magnetic field; 2) through a homogeneous medium with a gradient of ambient magnetic field strength. From the simulations, we synthesize the synchrotron radio emission, making different assumptions about the details of acceleration and injection of relativistic electrons.

Results. We find that asymmetric BSNRs are produced if the line-of-sight is not aligned with the gradient of ambient plasma density or with the gradient of ambient magnetic field strength. We derive useful parameters to quantify the degree of asymmetry of the remnants that may provide a powerful diagnostic of the microphysics of strong shock waves through the comparison between models and observations.

Conclusions. BSNRs with two radio limbs of different brightness can be explained if a gradient of ambient density or, most likely, of ambient magnetic field strength is perpendicular to the radio limbs. BSNRs with converging similar radio arcs can be explained if the gradient runs between the two arcs.
\end{abstract}

Key words. magnetohydrodynamics (MHD) - shock waves - ISM: supernova remnants - ISM: magnetic fields radio continuum: ISM

\section{Introduction}

It is widely accepted that the structure and the chemical abundances of the interstellar medium (ISM) are strongly influenced by supernova ( $\mathrm{SN}$ ) explosions and by their remnants (SNRs). However, the details of the interaction between SNR shock fronts and ISM depend, in principle, on many factors, among which the multiple-phase structure of the medium, its density and temperature, the intensity and direction of the ambient magnetic fields. These factors are not easily determined and this somewhat hampers our detailed understanding of the complex ISM.

The bilateral supernova remnants (BSNRs, Gaensler 1998; also called "barrel-shaped", Kesteven \& Caswell 1987, or "bipolar", Fulbright \& Reynolds 1990) are considered a benchmark for the study of large scale SNR-ISM interactions, since no small-scale effect like encounters with ISM clouds seems to be relevant. The BSNRs are characterized by two opposed radiobright limbs separated by a region of low surface brightness. In general, the remnants appear asymmetric, distorted and elongated with respect to the shape and surface brightness of the two opposed limbs. In most (but not all) of the BSNRs the symmetry axis is parallel to the galactic plane, and this has been interpreted as a difficulty for "intrinsic" models, e.g. models based on SN jets, rather than for "extrinsic" models, e.g. models based on properties of the surrounding galactic medium (Gaensler 1998).

In spite of the interest around BSNRs, a satisfactory and complete model which explains the observed morphology and the origin of the asymmetries does not exist. The galactic medium is supposed to be stratified along the lines of constant galactic latitude, and characterized by a large-scale ambient magnetic field with field lines probably mostly aligned with the galactic plane. The magnetic field plays a three-fold role: first, a magnetic tension and a gradient of the magnetic field strength is present where the field is perpendicular to the shock velocity leading to a compression of the plasma; second, cosmic ray acceleration is most rapid where the field lines are perpendicular to the shock speed (Jokipii 1987; Ostrowski 1988); third, the electron injection could be favored where the magnetic field is parallel to the shock speed (Ellison et al. 1995). Gaensler (1998) notes that magnetic models (i.e. those considering uniform ISM and ordered magnetic field) cannot explain the asymmetric morphology of most BSNRs, and invokes a dynamic model based on preexisting ISM inhomogeneities, e.g. large-scale density gradients, tunnels, and cavities. Unfortunately, the predictions of these adhoc models have consisted so far of a qualitative estimate of the BSNRs morphology, with no real estimates of the ISM density interacting with the shock. Also, non-uniform ambient magnetic fields most likely explain asymmetries in BSNRs, without having to assume ad-hoc density ISM structures. Two main aspects of the nature of BSNRs, therefore, remain unexplored: how and under which physical conditions do the asymmetries originate in BSNRs? What is more effective in determining the morphology and the asymmetries of this class of SNRs, the ambient magnetic field or the non-uniform ISM?

Answering such questions at an adequate level requires detailed physical modeling, high-level numerical implementations and extensive simulations. Our purpose here is to investigate 
whether the morphology of BSNR observed in the radio band could be mainly determined by the propagation of a shock through a non-uniform ISM or, rather, across a non-uniform ambient magnetic field. To this end, we model the propagation of a shock generated by an SN explosion in the magnetized nonuniform ISM with detailed numerical MHD simulations, considering two complementary cases of shock propagation: 1) through a gradient of ambient density with a uniform ambient magnetic field; 2) through a homogeneous isothermal medium with a gradient of ambient magnetic field strength.

In Sect. 2 we describe the MHD model, the numerical setup, and the synthesis of synchrotron emission; in Sect. 3 we analyze the effects the environment has on the radio emission of the remnant; finally in Sects. 4 and 5 we discuss the results and draw our conclusions.

\section{Model}

\subsection{Magnetohydrodynamic modeling}

We model the propagation of an SN shock front through a magnetized ambient medium. The model includes no radiative cooling, no thermal conduction, no eventual magnetic field amplification, and no effects on shock dynamics due to back-reaction of accelerated cosmic rays. The shock propagation is modeled by numerically solving the time-dependent ideal MHD equations of mass, momentum, and energy conservation in a 3D Cartesian coordinate system $(x, y, z)$ :

$$
\begin{aligned}
& \frac{\partial \rho}{\partial t}+\nabla \cdot(\rho \boldsymbol{u})=0, \\
& \frac{\partial \rho \boldsymbol{u}}{\partial t}+\nabla \cdot(\rho \boldsymbol{u} \boldsymbol{u}-\boldsymbol{B B})+\nabla P_{*}=0, \\
& \frac{\partial \rho E}{\partial t}+\nabla \cdot\left[\boldsymbol{u}\left(\rho E+P_{*}\right)-\boldsymbol{B}(\boldsymbol{u} \cdot \boldsymbol{B})\right]=0, \\
& \frac{\partial \boldsymbol{B}}{\partial t}+\nabla \cdot(\boldsymbol{u B}-\boldsymbol{B u})=0,
\end{aligned}
$$

where

$$
P_{*}=P+\frac{B^{2}}{2}, \quad E=\epsilon+\frac{1}{2}|\boldsymbol{u}|^{2}+\frac{1}{2} \frac{|\boldsymbol{B}|^{2}}{\rho},
$$

are the total pressure (thermal pressure, $P$, and magnetic pressure) and the total gas energy (internal energy, $\epsilon$, kinetic energy, and magnetic energy) respectively, $t$ is the time, $\rho=\mu m_{\mathrm{H}} n_{\mathrm{H}}$ is the mass density, $\mu=1.3$ is the mean atomic mass (assuming cosmic abundances), $m_{\mathrm{H}}$ is the mass of the hydrogen atom, $n_{\mathrm{H}}$ is the hydrogen number density, $\boldsymbol{u}$ is the gas velocity, $T$ is the temperature, and $\boldsymbol{B}$ is the magnetic field. We use the ideal gas law, $P=(\gamma-1) \rho \epsilon$, where $\gamma=5 / 3$ is the adiabatic index. The simulations are performed using the FLASH code (Fryxell et al. 2000), an adaptive mesh refinement multiphysics code for astrophysical plasmas.

As initial conditions, we adopted the model profiles of Truelove \& McKee (1999), assuming a spherical remnant with radius $r_{0 \mathrm{snr}}=4 \mathrm{pc}$ and with total energy $E_{0}=1.5 \times 10^{51} \mathrm{erg}$, originating from a progenitor star with mass of $15 M_{\text {sun }}$, and propagating through an unperturbed magnetohydrostatic medium. The initial total energy is partitioned so that $1 / 4$ of the SN energy is contained in thermal energy, and the other $3 / 4$ in kinetic energy. The explosion is at the center $(x, y, z)=(0,0,0)$ of the
Table 1. Relevant initial parameters of the simulations: $n_{0}$ and $n_{i}$ are particle number densities of the stratified unperturbed ISM (see text), $h$ is the density scale length, and $(x, y, z)$ are the coordinates of the magnetic dipole moment. The ambient medium is either uniform or with an exponential density stratification along the $x$ or the $z$ direction ( $x$-strat. and $z$-strat., respectively); the ambient magnetic field is uniform or dipolar with the dipole oriented along the $x$ axis and located at $(x, y, z)$.

\begin{tabular}{lcccccc}
\hline \hline & ISM & $\begin{array}{c}n_{0} \\
\mathrm{~cm}^{-3}\end{array}$ & $\begin{array}{c}n_{i} \\
\mathrm{~cm}^{-3}\end{array}$ & $\begin{array}{c}h \\
\mathrm{pc}\end{array}$ & $\boldsymbol{B}$ & $\begin{array}{c}(x, y, z) \\
\mathrm{pc}\end{array}$ \\
\hline GZ1 & $z$-strat. & 0.05 & 0.2 & 25 & uniform & - \\
GZ2 & $z$-strat. & 0.05 & 0.2 & 10 & uniform & - \\
GX1 & $x$-strat. & 0.05 & 0.2 & 25 & uniform & - \\
GX2 & $x$-strat. & 0.05 & 0.2 & 10 & uniform & - \\
DZ1 & uniform & 0.25 & - & - & $z$-strat. & $(0,0,-100)$ \\
DZ2 & uniform & 0.25 & - & - & $z$-strat. & $(0,0,-50)$ \\
DX1 & uniform & 0.25 & - & - & $x$-strat. & $(-100,0,0)$ \\
DX2 & uniform & 0.25 & - & - & $x$-strat. & $(-50,0,0)$ \\
\hline
\end{tabular}

computational domain which extends between -30 and $30 \mathrm{pc}$ in all directions. At the coarsest resolution, the adaptive mesh algorithm used in the FLASH code (PARAMESH; MacNeice et al. 2000) uniformly covers the 3D computational domain with a mesh of $8^{3}$ blocks, each with $8^{3}$ cells. We allow for 3 levels of refinement, with resolution increasing twice at each refinement level. The refinement criterion adopted (Löhner 1987) follows the changes in density and temperature. This grid configuration yields an effective resolution of $\approx 0.1 \mathrm{pc}$ at the finest level, corresponding to an equivalent uniform mesh of $512^{3}$ grid points. We assume zero-gradient conditions at all boundaries.

We follow the expansion of the remnant for $22 \mathrm{kyr}$, considering two sets of simulations: 1) through a gradient of ambient density with a uniform ambient magnetic field; or 2) through a homogeneous isothermal medium with a gradient of ambient magnetic field strength. Table 1 summarizes the physical parameters characterizing the simulations.

In the first set of simulations, the ambient magnetic field is assumed uniform with strength $\boldsymbol{B}=1 \mu \mathrm{G}$ and oriented parallel to the $x$ axis. The ambient medium is modeled with an exponential density stratification along the $x$ or the $z$ direction (i.e. parallel or perpendicular to the $\boldsymbol{B}$ field) of the form: $n(\xi)=n_{0}+n_{i} \exp (-\xi / h)$ (where $\xi$ is, respectively, $x$ or $z$ ) with $n_{0}=0.05 \mathrm{~cm}^{-3}$ and $n_{i}=0.2 \mathrm{~cm}^{-3}$, and where $h$ (set either to $25 \mathrm{pc}$ or to $10 \mathrm{pc}$ ) is the density scale length. This configuration has been used by e.g. Hnatyk \& Petruk (1999) to describe the SNR expansion in an environment with a molecular cloud. Our choice leads to a density variation of a factor $\sim 6$ or $\sim 60$, respectively, along the $x$ or the $z$ direction over the spatial domain considered (60 pc in total). The temperature of the unperturbed ISM is $T=10^{4} \mathrm{~K}$ at $\xi=0$ and is determined by pressure balance elsewhere. The adopted values of $T=10^{4} \mathrm{~K}, n=0.25 \mathrm{~cm}^{-3}$, and $B=1 \mu \mathrm{G}$ at $\xi=0$, outside the remnant, lead to $\beta \sim 17$ (where $\beta=P /\left(B^{2} / 8 \pi\right)$ is the ratio of thermal to magnetic pressure) a typical order of magnitude of $\beta$ in the diffuse regions of the ISM (Mac Low \& Klessen 2004).

In the second set of simulations, the unperturbed ambient medium is uniform with temperature $T=10^{4} \mathrm{~K}$ and particle number density $n=0.25 \mathrm{~cm}^{-3}$. The ambient magnetic field, $\boldsymbol{B}$, is assumed to be dipolar. This idealized situation is adopted here mainly to ensure magnetostaticity of the non-uniform field. The dipole is oriented parallel to the $x$ axis and located on the $z$ axis $(x=y=0)$ either at $z=-100 \mathrm{pc}$ or at $z=-50 \mathrm{pc}$; alternatively the dipole is located on the $x$ axis $(y=z=0)$ either at $x=-100 \mathrm{pc}$ or at $x=-50 \mathrm{pc}$ (as shown in Fig. 1). 


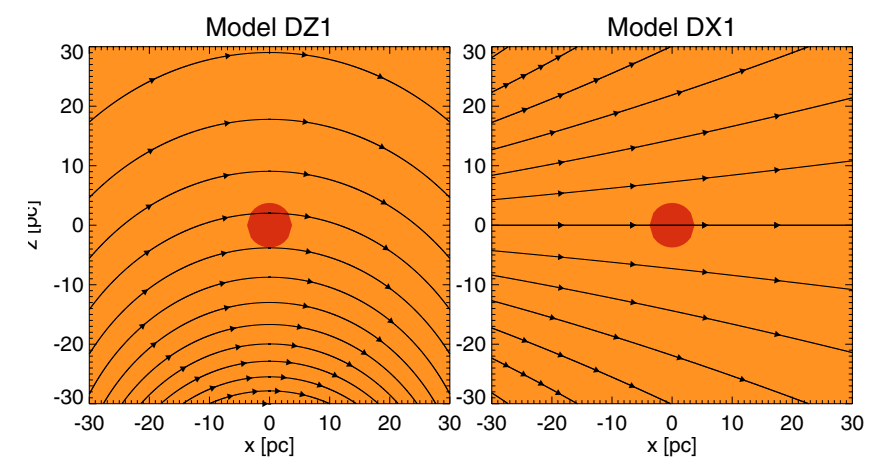

Fig. 1. 2-D sections in the $(x, z)$ plane of the initial mass density distribution and initial configuration of the unperturbed dipolar ambient magnetic field in two cases: dipole moment located on the $z$ axis (DZ1, left panel), or on the $x$ axis (DX1, right panel). The initial remnant is at the center of the domain. Black lines are magnetic field lines.

In both configurations, the field strength varies by a factor $\sim 6(z$ or $x=-100 \mathrm{pc}$ ) or $\sim 60$ ( $z$ or $x=-50 \mathrm{pc}$ ) over $60 \mathrm{pc:}$ in the first case in the direction perpendicular to the average ambient field $\langle\boldsymbol{B}\rangle$, whereas in the second case parallel to $\langle\boldsymbol{B}\rangle$. In all the cases, the initial magnetic field strength is set to $B=1 \mu \mathrm{G}$ at the center of the $\mathrm{SN}$ explosion $(x=y=z=0)$.

Note that the transition time from adiabatic to radiative phase for a SNR is (e.g. Blondin et al. 1998; Petruk 2005)

$t_{\text {tr }}=2.84 \times 10^{4} E_{51}^{4 / 17} n_{\text {ism }}^{-9 / 17} \mathrm{yr}$,

where $E_{51}=E_{0} /\left(10^{51} \mathrm{erg}\right)$ and $n_{\text {ism }}$ is the particle number density of the ISM. In our set of simulations, runs GZ2 and GX2 present the lowest values of the transition time, namely $t_{\mathrm{tr}} \approx 25 \mathrm{kyr}$. Since we follow the expansion of the remnant for $22 \mathrm{kyr}$, our modeled SNRs are in the adiabatic phase.

\subsection{Nonthermal electrons and synchrotron emission}

We synthesize the radio emission from the remnant, assuming that it is only due to synchrotron radiation from relativistic electrons distributed with a power law spectrum $N(E)=K E^{-\zeta}$, where $E$ is the electron energy, $N(E)$ is the number of electrons per unit volume with arbitrary directions of motion and with energies in the interval $[E, E+\mathrm{d} E], K$ is the normalization of the electron distribution, and $\zeta$ is the power law index. Following Ginzburg \& Syrovatskii (1965), the radio emissivity can be expressed as:

$i(v)=C_{1} K B_{\perp}^{\alpha+1} v^{-\alpha}$,

where $C_{1}$ is a constant, $B_{\perp}$ is the component of the magnetic field perpendicular to the line-of-sight $(\operatorname{LoS}), v$ is the frequency of the radiation, and $\alpha=(\zeta-1) / 2$ is the synchrotron spectral index (assumed to be uniform everywhere and taken as 0.5 as observed in many BSNRs). To compute the total radio intensity (Stokes parameter $I$ ) at a given frequency $v_{0}$, we integrate the emissivity $i\left(v_{0}\right)$ along the LoS:

$I\left(v_{0}\right)=\int i\left(v_{0}\right) \mathrm{d} l$

where $\mathrm{d} l$ is the increment along the LoS.

The normalization of the electron distribution $K_{\mathrm{s}}$ in Eq. (6) (index "s" refers to the immediately post-shock values) depends on the injection efficiency (the fraction of electrons that move into the cosmic-ray pool). Unfortunately, it is unknown how the injection efficiency evolves in time. On theoretical grounds, $K_{\mathrm{s}}$ is expected to vary with the shock velocity $V_{\mathrm{sh}}(t)$ and, in case of inhomogeneous ISM, with the immediately post-shock value of mass density, $\rho_{\mathrm{s}}$; let us assume that approximately $K_{\mathrm{s}} \propto \rho_{\mathrm{s}} V_{\mathrm{sh}}(t)^{-b}$. Reynolds (1998) considered three empirical alternatives for $b$ as a free parameter, namely, $b=0,-1,-2$. Petruk \& Bandiera (2006) showed that one can expect $b>0$ and its value could be $b \approx 4$. Stronger shocks are more successful in accelerating particles. To be accelerated effectively, in each Fermi cycle a particle should obtain a larger increase in momentum, which is proportional to the shock velocity. Negative $b$ reflects an expectation that injection efficiency may behave in a way similar to acceleration efficiency: stronger shocks might inject particles more effectively. In contrast, positive $b$ represents a different point of view: efficiencies of injection and acceleration may have opposite dependencies on the shock velocity. Stronger shocks produce higher turbulence which is expected to prevent more thermal particles from recrossing the shock from downstream to upstream and, therefore, from being injected. Since the picture of injection is quite unclear from both theoretical and observational points of view, we do not pay attention to the physical motivations of the value of $b$. Instead, our goal is to see how different trends in evolution of injection efficiency may affect the visible morphology of SNRs. Such understanding could be useful for future observational tests on the value of $b$.

We found, in agreement with Reynolds (1998), that the value of $b$ does not affect the main features of the surface brightness distribution if SNR evolves in uniform ISM. Therefore we use the value $b=0$ to produce the SNR images in models with uniform ISM (models DZ1, DZ2, DX1, and DX2). In cases where non-uniformity of ISM causes variation of the shock velocity in SNR (models GZ1, GZ2, GX1, and GX2), we calculate images for $b=-2,0,2$. We follow the model of Reynolds (1998) in describing the post-shock evolution of relativistic electrons. Adopting this approach and considering that $\zeta=2$ (being $\alpha=0.5$, see above), one obtains that (see Appendix A)

$$
\frac{K(a, t)}{K_{\mathrm{s}}(R, t)}=\left(\frac{P(a, t)}{P(R, t)}\right)^{-b / 2}\left(\frac{\rho_{\mathrm{o}}(a)}{\rho_{\mathrm{o}}(R)}\right)^{-(b+1) / 3}\left(\frac{\rho(a, t)}{\rho(R, t)}\right)^{5 b / 6+4 / 3}
$$

where $a$ is the lagrangian coordinate, $R$ is the shock radius, $\rho$ is the gas density, $P$ is the gas pressure, and the index "o" refers to the pre-shock values. It is important to note that this formula accounts for variation of injection efficiency caused by the nonuniformity of ISM.

The electron injection efficiency may also vary with the obliquity angle between the external magnetic field and the shock normal, $\phi_{\mathrm{Bn}}$. The numerical simulations suggest that injection efficiency is larger for parallel shocks, i.e. where the magnetic field is parallel to the shock speed (obliquity angle close to zero; Ellison et al. 1995). However, it has been shown (Fulbright \& Reynolds 1990) that models with injection strongly favoring parallel shocks produce SNR maps that do not resemble any known objects (it is also claimed that injection is more efficient where the magnetic field is perpendicular to the shock speed; Jokipii 1987). On the other hand, comparison of known SNRs morphologies with model SNR images calculated for different strengths of the injection efficiency dependence on obliquity suggests that the injection efficiency in real SNRs could not depend on obliquity (Petruk, in preparation). In such an unclear situation, we consider the three cases: quasi-parallel, quasi-perpendicular, and isotropic injection models. Following Fulbright \& Reynolds (1990), we model quasi-parallel injection 
by multiplying the normalization of the electron distribution $K$ by $\cos ^{2} \phi_{\mathrm{Bn} 2}$ (see also Leckband et al. 1989), where $\phi_{\mathrm{Bn} 2}$ is the angle between the shock normal and the post-shock magnetic field $^{1}$. By analogy with the quasi-parallel case, we model quasiperpendicular injection by multiplying $K$ by $\sin ^{2} \phi_{\mathrm{Bn} 2}$.

An important point is the degree of ordering of magnetic field downstream of the shock. Radio polarization observations of a number of SNRs (e.g. Tycho Dickel et al. 1991, SN1006 Reynolds \& Gilmore 1993) show the low degree of polarization, $10-15 \%$ (in case of ordered magnetic field the value expected is about 70\%; Fulbright \& Reynolds 1990), indicating a highly disordered magnetic field. Thus we calculate the synchrotron images of SNR for two opposite cases. First, since our MHD code gives us the three components of magnetic fields, we are able to calculate images with ordered magnetic fields. Second, we introduce the procedure of the magnetic field disordering (with randomly oriented magnetic field vector with the same magnitude in each point) and then synthesize the radio maps. In models which have a disordered magnetic field, we use the post-shock magnetic field before disordering to calculate the angle $\phi_{\mathrm{Bn} 2}$; as discussed by Fulbright \& Reynolds (1990), this corresponds to the assumption that the disordering process takes place over a longer time-scale than the electron injection, which occurrs in the close proximity of the shock. Since we found that the asymmetries induced by gradients either of ambient plasma density or of ambient magnetic field strength are not significantly affected by the degree of ordering of the magnetic field downstream of the shock, in the following we will focus on the models with a disordered magnetic field.

The goal of this paper is to look at whether non-uniform ISM or non-uniform magnetic fields can produce asymmetries on BSNRs morphology. In order to clearly see the role of these two factors in determining the morphology of BSNRs, we use some simplifying assumptions about electron kinetics and the behavior of magnetic fields in the vicinity of the shock front. Our calculations are performed in the test-particle limit, i.e. they ignore the energy in cosmic rays. In particular, we do not consider the possible amplification of magnetic fields by the cosmic-ray streaming instability (Lucek \& Bell 2000; Bell \& Lucek 2001). We expect that the main features of the modeled SNR morphology will not change if this process is independent of obliquity angle. If future investigations show without a doubt that magnetic field amplification varies strongly with obliquity, the role of this effect in producing BSNRs will have to be studied.

\section{Results}

In all the models examined, we found the typical evolution of adiabatic SNRs expanding through an organized ambient magnetic field (see Balsara et al. 2001 and references therein): the fast expansion of the shock front with temperatures of a few millions degrees, and the development of Richtmyer-Meshkov (R-M) instability, as the forward and reverse shocks progress through the ISM and ejecta, respectively (see Kane et al. 1999). As examples, Fig. 2 shows 2D sections in the $(x, z)$ plane of the distributions of mass density and of magnetic field strength for the models GZ2, DZ2, and DX2 at $t=18 \mathrm{kyr}$. The inner shell is dominated by the R-M instability that causes the plasma mixing

${ }^{1}$ For a shock compression ratio of 4 (the shock Mach number is $\gg 10$ in all directions during the whole evolution in each of our simulations), the obliquity angle between the external magnetic field and the shock normal, $\phi_{\mathrm{Bn}}$, is related to $\phi_{\mathrm{Bn} 2}$ by $\sin ^{2} \phi_{\mathrm{Bn} 2}=\left(\cot ^{2} \phi_{\mathrm{Bn}} / 16+1\right)^{-1}($ e.g. Fulbright \& Reynolds 1990).
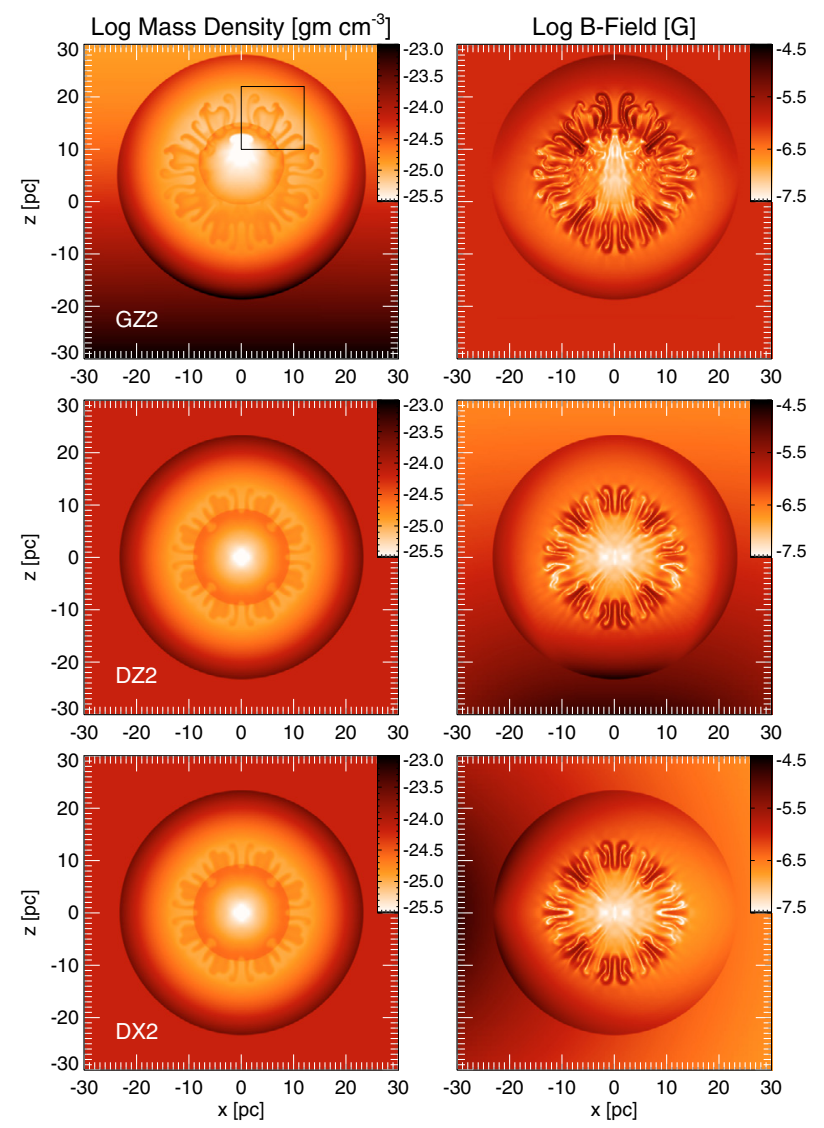

Fig. 2. 2-D sections in the $(x, z)$ plane of the mass density distribution (left panels), in log scale, and of the distribution of the magnetic-field strength (right panels), in log scale, in the simulations GZ2 (upper panels), DZ2 (middle panels), and DX2 (lower panels) at $t=18 \mathrm{kyr}$. The box in the upper left panel marks the region shown in Fig. 3.

and the magnetic field amplification. In the inner shell, the magnetic field shows a turbulent structure with preferentially radial components around the R-M fingers (see Fig. 3). Note that some authors have invoked the R-M instabilities to explain the dominant radial magnetic field observed in the inner shell of SNRs (e.g. Jun \& Norman 1996); however, in our simulations, the radial tendency is observed well inside the remnant and not immediately behind the shock as inferred from observations.

We found that, throughout the expansion, the shape of the remnant is not appreciably distorted by the ambient magnetic field because, for the values of explosion energy and ambient field strength (typical of SNRs) used in our simulations, the kinetic energy of the shock is many orders of magnitude larger than the energy density in the ambient $\boldsymbol{B}$ field (see also Mineshige \& Shibata 1990). The shape of the remnant does not differ visually from a sphere also in the cases with density stratification of the ambient medium ${ }^{2}$ (see Hnatyk \& Petruk 1999).

The radio emission of the evolved remnants is characterized by an incomplete shell morphology when the viewing angle is not aligned with the direction of the average ambient magnetic field (cf. Fulbright \& Reynolds 1990); in general, the radio emission shows an axis of symmetry with low levels of emission along it, and two bright limbs (arcs) on either side (see

2 In these cases, the remnant appears shifted toward the low density region; see upper panels in Fig. 2 (see also Dohm-Palmer \& Jones 1996). 


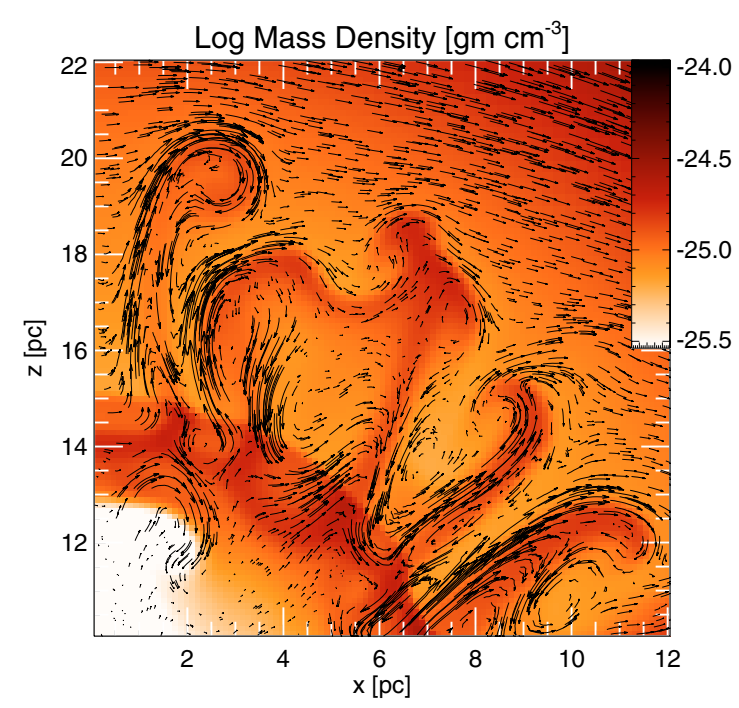

Fig. 3. Close-up view of the region marked with a box in Fig. 2. The dark fingers mark the R-M instability. The magnetic field is described by the superimposed arrows the length of which is proportional to the magnitude of the field vector.

also Gaensler 1998). This morphology is very similar to that observed in BSNRs.

\subsection{Obliquity angle dependence}

For each of the models listed in Table 1, we synthesized the synchrotron radio emission, considering each of the three cases of variation of electron injection efficiency with shock obliquity: quasi-parallel, quasi-perpendicular, and isotropic particle injection. As an example, Fig. 4 shows the synchrotron radio emission synthesized from the uniform ISM model DZ1 with randomized internal magnetic field at $t=18 \mathrm{kyr}$ in each of the three cases. We recall that for these uniform density cases, we have adopted an injection efficiency independent from the shock speed $(b=0$, Sect. 2.2). All images are maps of total intensity normalized to the maximum intensity of each map and have a resolution of 400 beams per remnant diameter $\left(D_{\mathrm{SNR}}\right)$. The images are derived when the LoS is parallel to the average direction of the unperturbed ambient magnetic field $\langle\boldsymbol{B}\rangle$ (LoS aligned with the $x$ axis), or perpendicular both to $\langle\boldsymbol{B}\rangle$ and to the gradient of field strength (LoS along $y$ ), or parallel to the gradient of field strength (LoS along $z$ ).

The different particle injection models produce images that can differ considerably in appearance. In particular, the quasiparallel case leads to morphologies of the remnant not reproduced by the other two cases: a center-brightened SNR when the LoS is aligned with $x$ (top left panel in Fig. 4), a BSNR with two bright arcs slanted and converging on the side where $\boldsymbol{B}$ field strength is higher when the LoS is along $y$ (top center panel), and a remnant with two symmetric bright spots located between the center and the border of the remnant when the LoS is along $z$ (top right panel). Neither the center-brightened remnant nor the double peak structure, showing no structure describable as a shell, seems to be observed in SNRs ${ }^{3}$. We found analogous morphologies in all the models listed in Table 1, considering the quasiparallel case. As extensively discussed by Fulbright \& Reynolds (1990) for models with uniform ambient magnetic field and

3 Excluding filled center and composite SNRs, but these are due to energy input from a central pulsar. $b=-2$, we also conclude that the quasi-parallel case leads to radio images unlike any observed SNR (see also Kesteven \& Caswell 1987).

The isotropic case leads to remnant morphologies similar to those produced in the quasi-perpendicular case, although the latter case shows deeper minima in the radio emission than the first one. When the LoS is aligned with $x$ (middle left and bottom left panels in Fig. 4) or with $y$ (middle center and bottom center panels), the remnants have one bright arc on the side where the $\boldsymbol{B}$ strength is higher. When the LoS is aligned with $z$ (middle right and bottom right panels), the remnants have two opposed arcs that appear perfectly symmetric. We found that the isotropic and quasi-perpendicular cases lead to morphologies of the remnants similar to those observed.

\subsection{Non-uniform ISM: dependence from parameter $b$}

For models describing the SNR expansion through a nonuniform ISM (models GZ1, GZ2, GX1, GX2), we derived the synthetic radio maps considering three alternatives for the dependence of the injection efficiency on the shock speed, namely $b=-2,0,2$ (see Sect. 2.2). As an example, Fig. 5 shows the synthetic maps derived from model GZ1 with a randomized internal magnetic field, assuming quasi-perpendicular particle injection, and considering $b=-2$ (top panels), $b=0$ (middle), and $b=2$ (bottom).

When the LoS is not aligned with the density gradient, the radio images show asymmetric morphologies of the remnants. In this case, the main effect of varying $b$ is to change the degree of asymmetry observed in the radio maps. In the example shown in Fig. 5, the density gradient is aligned with the $z$ axis and asymmetric morphologies are produced when the LoS is aligned with $x$ (left panels) or with $y$ (center panels). In all the cases, the remnant is brighter where the mass density is higher. On the other hand, the degree of asymmetry increases with increasing value of $b$.

The reason for such behavior consists in the balance between the roles of the shock velocity and of density in changing the injection efficiency. Consider, as an example, the top left panel in Fig. 5: the increase of the shock velocity on the north (due to fall of the ambient density) leads to an increase in the brightness there (due to rise of the injection efficiency) that partially balances the increase in the brightness on the south due to higher density of ISM. On the other hand, for the model shown in the bottom left panel in Fig. 5, the fraction of accelerated electrons increases on the south due to both the rise of density and the decrease in the shock velocity.

When the LoS is aligned with the density gradient, the radio images are symmetric. In the example shown in Fig. 5, this corresponds to the maps derived when the LoS is along $z$ (right panels); the remnants are characterized by two opposed arcs with identical surface brightness.

\subsection{Morphology}

Figure 6 shows the radio emission maps, at a time of $18 \mathrm{kyr}$, synthesized from models with a gradient of ambient plasma density (panels A and D; assuming $b=2$ ) and of ambient $\boldsymbol{B}$ field strength (panels B and E; assuming $b=0$ ). All the models assume quasi-perpendicular particle injection (the isotropic case produces radio maps with similar morphologies and the quasi-parallel case is discussed later) and randomized internal magnetic field. The viewing angle is perpendicular both to the 

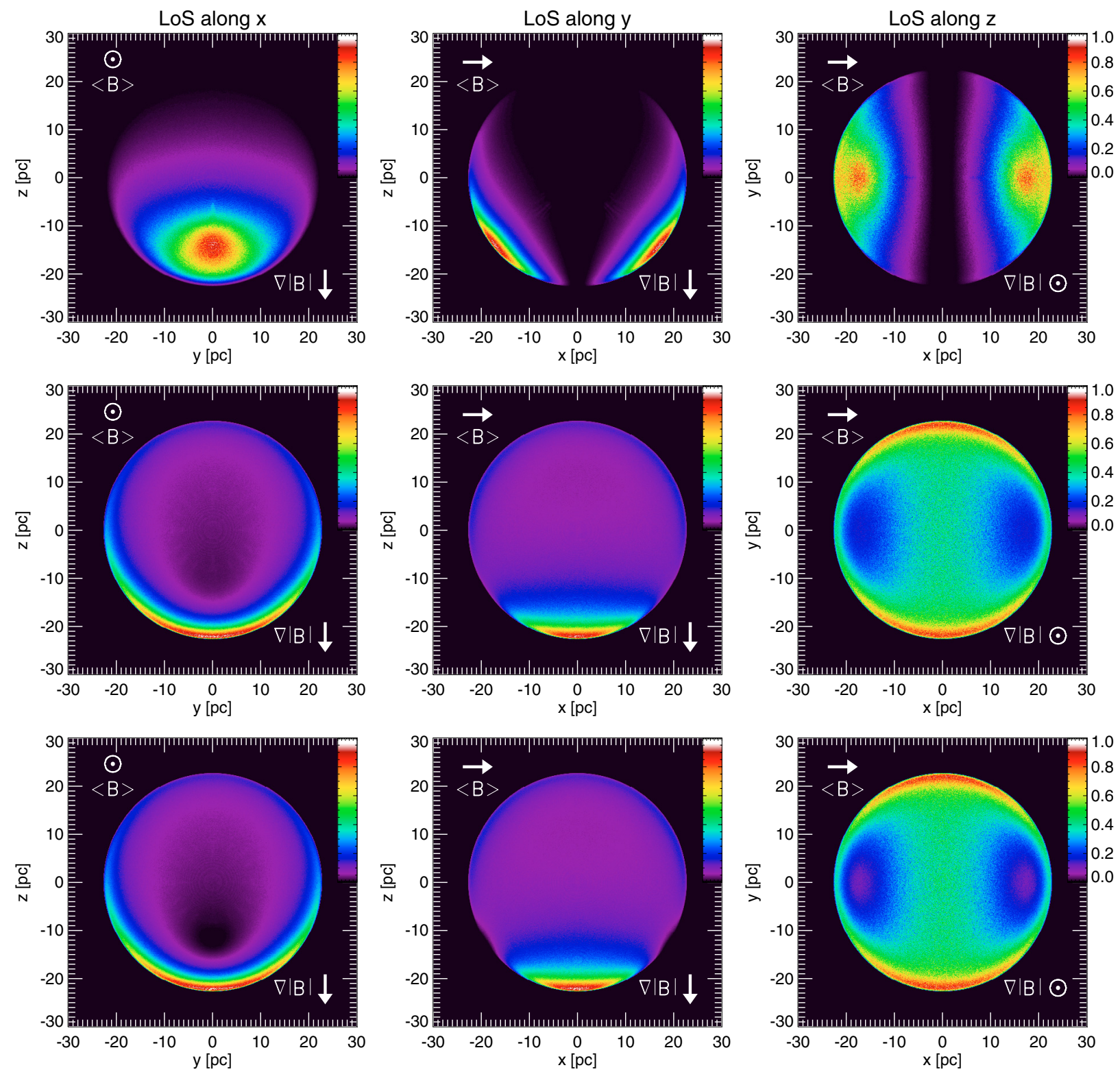

Fig. 4. Synchrotron radio emission (normalized to the maximum of each panel), at $t=18 \mathrm{kyr}$, synthesized from model DZ1 assuming $b=0$ (see text) and randomized internal magnetic field, when the LoS is aligned with the $x$ (left), $y$ (center), or $z$ (right) axis. The figure shows the quasi-parallel (top), isotropic (middle), and quasi-perpendicular (bottom) particle injection cases. The color scale is linear and is given by the bar on the right. The directions of the average unperturbed ambient magnetic field, $\langle\boldsymbol{B}\rangle$, and of the magnetic field strength gradient, $\nabla|\boldsymbol{B}|$, are shown in the upper left and lower right corners of each panel, respectively.

average direction of the unperturbed ambient magnetic field, $\langle\boldsymbol{B}\rangle$, (direct along the $x$ axis) and to the gradients of density or field strength (direct either along $z$, panels $\mathrm{A}$ and $\mathrm{B}$, or $x$, panels $\mathrm{D}$ and $\mathrm{E}$ ). The right panels show examples of the radio maps of the SNRs G338.1+04 (panel C, data from Whiteoak \& Green 1996) and G296.5+10.0 (panel F, from Gaensler 1998).

In the quasi-perpendicular case discussed here, the maximum synchrotron emissivity is reached where the magnetic field is strongly compressed. This configuration has been referred to as "equatorial belt" (e.g. Rothenflug et al. 2004); $\langle\boldsymbol{B}\rangle$ runs between the two opposed arcs (along the $x$ axis). We found that, when the density or the magnetic field strength gradient is perpendicular to the field itself, the morphology of the radio map strongly depends on the viewing angle. In these cases, the two opposed arcs appear perfectly symmetric when the $\operatorname{LoS}$ is aligned with the gradient (see, for instance, the right panels in Fig. 5), otherwise the two arcs can have a very different radio brightness, leading to strongly asymmetric BSNRs (see panels A and B in Fig. 6). In the former case (LoS aligned with the gradient), the remnant is characterized by two axes of symmetry: one between the two symmetric arcs and the other perpendicular to the two. In models with strong magnetic field strength gradients (DZ2; $\boldsymbol{B}$ varies by a factor $\sim 60$ over $60 \mathrm{pc}$ ), we found that the radio images are center-brightened when the LoS is aligned with the gradient (figure not reported). The fact that center-brightened remnants are not observed suggests that the external $\boldsymbol{B}$ varies moderately in the neighborhood of the remnants.

In case of asymmetry, the gradient is always perpendicular to the arcs, and the brightest arc is located where either magnetic field strength or plasma density is higher (see panels A 

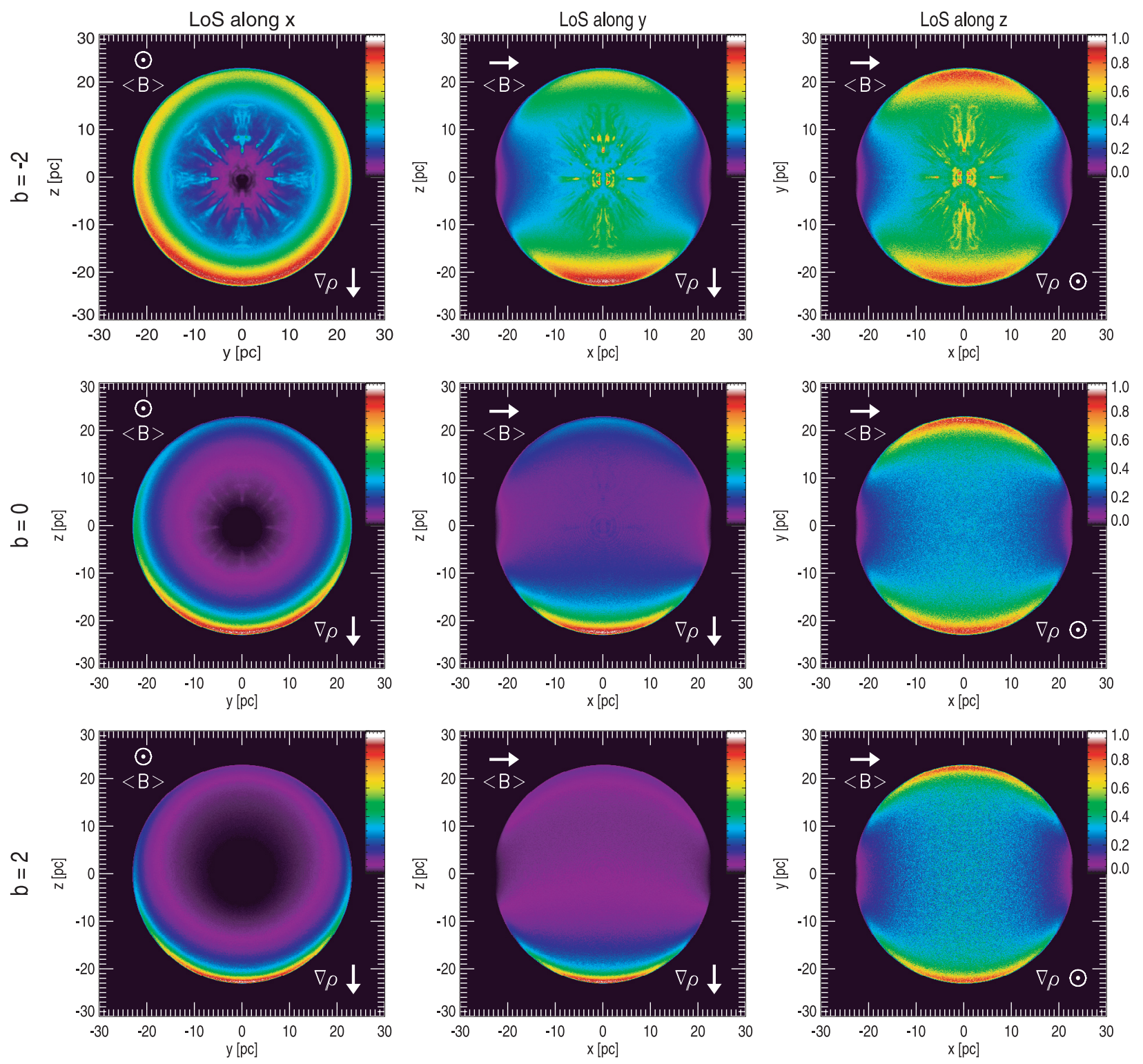

Fig. 5. Presentation as in Fig. 4 for model GZ1 with randomized internal magnetic field, assuming quasi-perpendicular particle injection and $b=-2$ (top panels), $b=0$ (middle), and $b=2$ (bottom). The directions of the average unperturbed ambient magnetic field, $\langle\boldsymbol{B}\rangle$, and of the ambient plasma density gradient, $\nabla \rho$, are shown in the upper left and lower right corners of each panel, respectively.

and B in Fig. 6), since the synchrotron emission depends on the plasma density, on the pressure, and on the field strength (see Eqs. (6) and (8)); in this case, there is only one axis of symmetry oriented along the density or $\boldsymbol{B}$ gradient. When the LoS is parallel to $\langle\boldsymbol{B}\rangle$ (along $x$ in our models), the radio maps show a shell structure with a maximum intensity located where magnetic field strength or plasma density is higher (see left panels in Fig. 4 for isotropic and quasi-perpendicular cases and left panels in Fig. 5). Our simulations show that, when the density or the magnetic field strength gradient is perpendicular to the field itself, remnants with a monopolar morphology can be observed at LoS not aligned with the gradient (see also Reynolds \& Fulbright 1990). Examples of observed monopolar remnants are G338.1+0.4 (see panel C in Fig. 6) or G327.4+1.0 or G341.9-0.3.

When the density or $\boldsymbol{B}$ field strength gradient is parallel to $\langle\boldsymbol{B}\rangle$ (panels D and E in Fig. 6) and the LoS lies in the plane perpendicular to $\langle\boldsymbol{B}\rangle$, the morphology of the radio map does not depend on the viewing angle and the two opposed arcs have the same radio brightness. In these cases, however, there is only one axis of symmetry and the two arcs appear slanted and converging on the side where field strength or plasma density is higher; again, the symmetry axis is aligned with the density or $\boldsymbol{B}$ strength gradient. Examples of these kinds of objects are G296.5+10.0 (see panel F in Fig. 6) or G332.4-004 or SN1006 (which is, however, much younger than the simulated SNRs). When the external magnetic field is parallel to the LoS, because the system is symmetric about the magnetic field, the remnant is axially symmetric and the radio maps show a complete radio shell at constant intensity.

In the quasi-parallel case, $\langle\boldsymbol{B}\rangle$ runs across the arcs. This configuration has been referred to as "polar caps" and it has been invoked for the SN1006 remnant (Rothenflug et al. 2004). The quasi-parallel case, apart from the center-brightened morphology discussed in Sect. 3.1, can also produce remnant morphologies similar to those shown in Fig. 6. As examples, Fig. 7 shows the radio emission maps obtained in the cases discussed in Fig. 6, but assuming quasi-parallel instead of quasi-perpendicular particle injection. Again, the viewing angle is perpendicular both to 

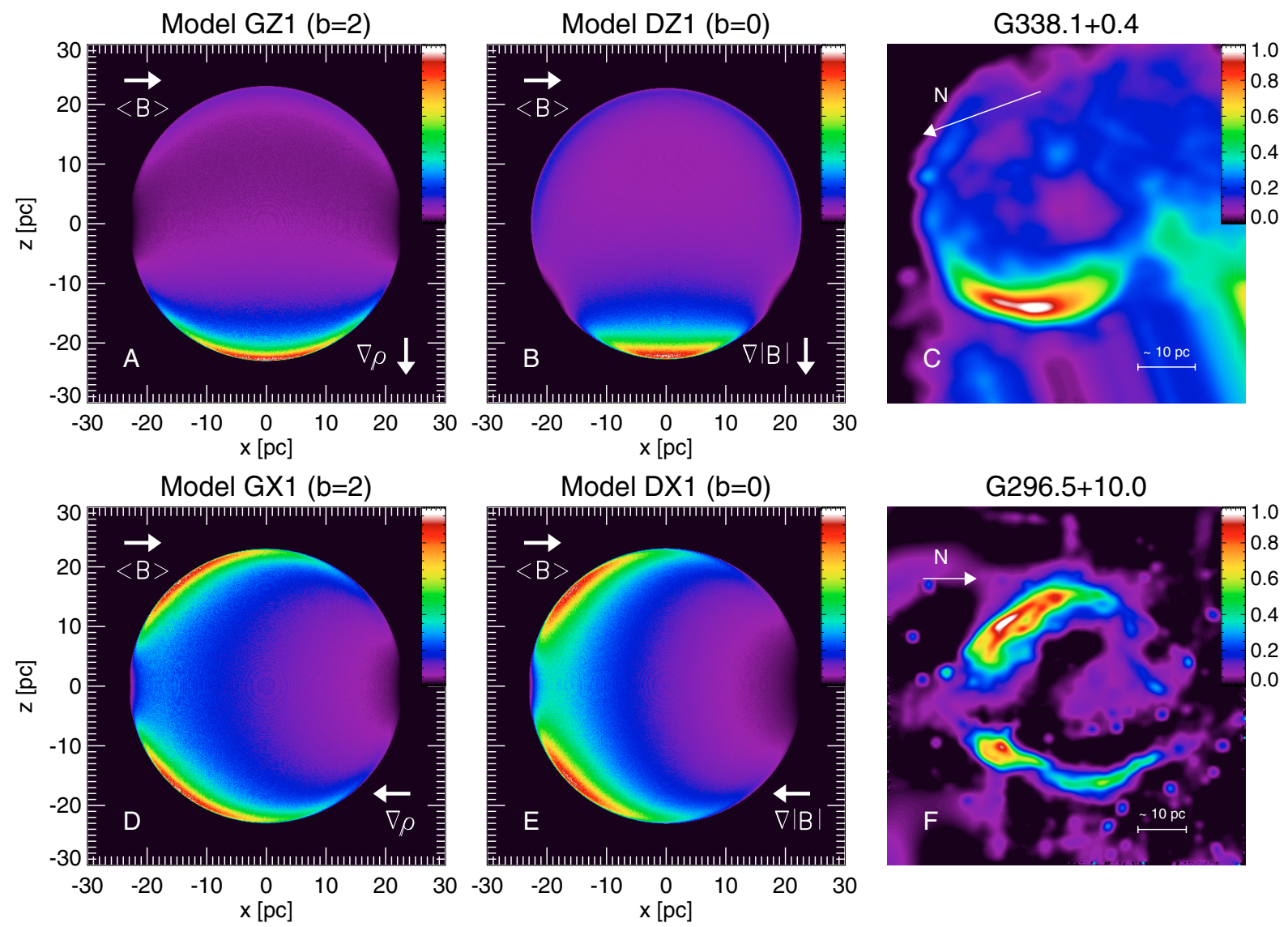

Fig. 6. Synchrotron radio emission (normalized to the maximum of each panel), at $t=18 \mathrm{kyr}$, synthesized from models assuming a gradient of ambient plasma density (panels $\mathbf{A}$ ) and $\mathbf{D}$ ); with $b=2$ ) or of ambient magnetic field strength (panels $\mathbf{B}$ ) and $\mathbf{E}$ ); with $b=0$ ) when the LoS is aligned with the $y$ axis. All the models assume quasi-perpendicular particle injection. The directions of the average unperturbed ambient magnetic field, $\langle\boldsymbol{B}\rangle$, and of the plasma density or magnetic field strength gradient, are shown in the upper left and lower right corners of each panel, respectively. The right panels show two examples of radio maps (data adapted from Whiteoak \& Green 1996 and Gaensler 1998; the arrows point in the north direction) collected for the SNRs G338.1+0.4 (panel C)) and G296.5+10.0 (panel F)). The color scale is linear and is given by the bar on the right.

$\langle\boldsymbol{B}\rangle$ (direct along the $x$ axis) and to the gradients of density or field strength (direct either along $z$, panels $\mathrm{A}$ and $\mathrm{B}$, or $x$, panels $C$ and D). In the quasi-parallel case, remnants with a bright radio limb are produced if the gradient of ambient density or of ambient $\boldsymbol{B}$ field strength is parallel to $\langle\boldsymbol{B}\rangle$ (instead of perpendicular to $\langle\boldsymbol{B}\rangle$ as in the quasi-perpendicular case), whereas slanting similar radio arcs are obtained if the gradient is perpendicular to $\langle\boldsymbol{B}\rangle$ (instead of parallel as in the quasi-perpendicular case).

\section{Discussion}

Our simulations show that asymmetric BSNRs are explained if the ambient medium is characterized by gradients either of density or of ambient magnetic field strength: the two opposed arcs have different surface brightness if the gradient runs across the arcs (see panels A and B in Fig. 6, and panels C and D in Fig. 7), whereas the two arcs appear slanted and converging on one side if the gradient runs between them (see panels D and E in Fig. 6 and panels $\mathrm{A}$ and $\mathrm{B}$ in Fig. 7). In all the cases (including the three alternatives for the particle injection), the symmetry axis of the remnant is always aligned with the gradient.

From the radio maps, we derived the azimuthal intensity profiles: we first find the point on the map where the intensity is maximum; then the contour of points at the same distance from the center of the remnant as the point of maximum intensity defines the azimuthal radio intensity profile. Following Fulbright \& Reynolds (1990), we quantify the degree of "bipolarity" of the remnants by using the so-called azimuthal intensity ratio $A$, i.e. the ratio of maximum to minimum intensity derived from the azimuthal intensity profiles. In addition, we quantify the degree of asymmetry of the BSNRs by using a measure we call the azimuthal intensity ratio $R_{\max } \geq 1$, i.e. the ratio of the maxima of intensity of the two limbs as derived from the azimuthal intensity profiles, and the azimuthal distance $\theta_{\mathrm{D}}$, i.e. the distance in deg of the two maxima. In the case of symmetric BSNRs, $R_{\max }=1$ and $\theta_{\mathrm{D}}=180^{\circ}$. As already noted by Fulbright $\&$ Reynolds (1990), the parameter $A$ depends on the spatial resolution of the radio maps and on the aspect angle (i.e. the angle between the LoS and the unperturbed magnetic field); moreover we note that, in real observations, the measure of $A$ gives a lower limit to its real value if the background is not accurately taken into account. On the other hand, the parameters $R_{\max }$ and $\theta_{\mathrm{D}}$ have a much less critical dependency on these factors and, therefore, they may provide a more robust diagnostic in the comparison between models and observations.

Figure 8 shows the values of $A, R_{\max }$, and $\theta_{\mathrm{D}}$ derived for all the cases examined in this paper, considering the LoS aligned with the $y$ axis, and radio maps with a resolution of 25 beams 

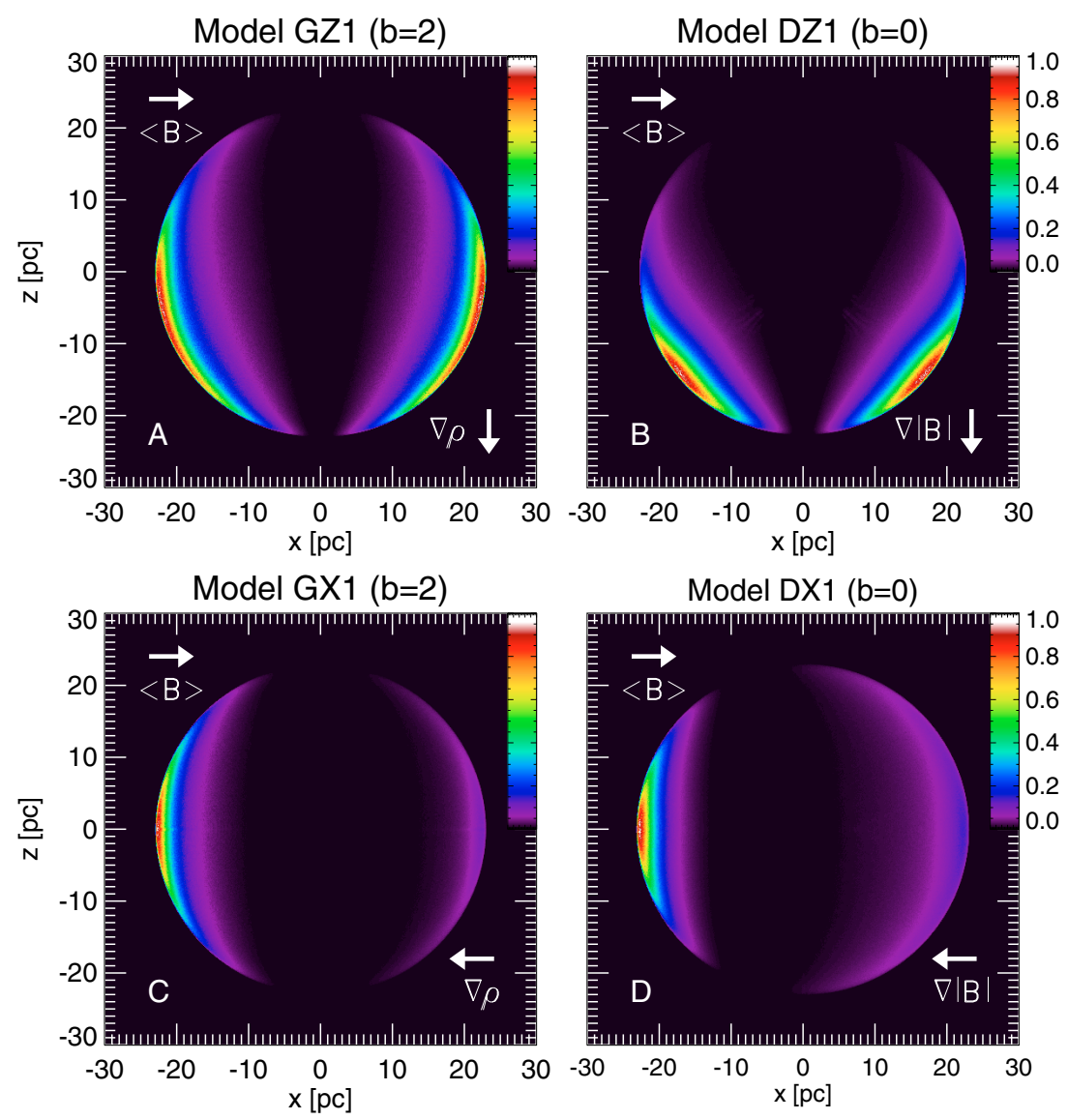

Fig. 7. Presentation as in Fig. 6, assuming quasi-parallel instead of quasi-perpendicular particle injection.

per remnant diameter ${ }^{4}\left(D_{\mathrm{SNR}}\right)$. Note that our choice of the LoS aligned with $y$ (aspect angle $\phi=90^{\circ}$ ) implies that the values of $A$ in Fig. 8 are upper limits, being $A$ maximum at $\phi=90^{\circ}$ and minimum at $\phi=0^{\circ}$ (see Fulbright \& Reynolds 1990). The three models of particle injection (isotropic, quasi-perpendicular, and quasi-parallel) lead to different values of $A$. In the isotropic and quasi-perpendicular cases, most of the values of $A$ range between 5 and 20 (for model DX2, $A$ is even larger than 100); in the quasi-parallel case, the values of $A$ are larger than 500.

We found that, in general, a gradient of the ambient magnetic field strength leads to remnant morphologies similar to those induced by a gradient of plasma density (compare, for instance, panel A with B and panel D with E in Fig. 6). On the other hand, if $b<0$ in GX and GZ models, ambient $\boldsymbol{B}$ field gradients are more effective in determining the morphology of asymmetric BSNRs. This is seen in a more quantitative form in Fig. 8. DX and DZ models give $R_{\max }$ values higher and $\theta_{\mathrm{D}}$ values lower than GX and GZ models with $b<0$ : a modest gradient of the magnetic field (models DX1 and DZ1) gives a value of $R_{\max }$ higher or $\theta_{\mathrm{D}}$ lower than the two models with strong density gradients (models GX2 and GZ2) and $b<0$.

Figure 8 also shows that, in models with a density gradient, the degree of asymmetry of the remnant increases with increasing value of $b$; the GX and GZ models with $b>0$ give values of $R_{\max }$ and $\theta_{\mathrm{D}}$ comparable with (or, in the case of $R_{\max }$, even larger than) those derived from DX and DZ models. In the case of quasi-parallel particle injection for remnants with converging similar arcs, it is necessary to have a strong gradient of density

4 After the radio maps are calculated, they are convolved with a Gaussian function with $\sigma$ corresponding to the required resolution. perpendicular to $\boldsymbol{B}$ and $b \geq 0$ (compare models GZ1 and GZ2 in the lower panel in Fig. 8) to give values of $\theta_{\mathrm{D}}$ comparable to those obtained with a moderate gradient of ambient $\boldsymbol{B}$ field strength perpendicular to $\boldsymbol{B}$ (see model DZ1 in Fig. 8).

In order to compare our model predictions with observations of real BSNRs, we have selected 11 SNR shells which show one or two clear lobes of emission in archive total intensity radio images, separated by a region of minima. We have discarded all those cases in which several point-like or extended sources appear superimposed to the bright limbs, or other cases in which the location of maximum or minimum emission around the shell is difficult to derive. Unlike other lists of BSNRs published in the literature (e.g. Kesteven \& Caswell 1987; Fulbright \& Reynolds 1990; Gaensler 1998), here we focus on a reliable measure of the parameters $A, R_{\max }$, and $\theta_{\mathrm{D}}$; we avoid, therefore, patchy and irregular limbs, as in the case of G320.4-01.2 of Gaensler (1998). Moreover, we are obviously not discarding remnants which have constraints on $A, R_{\max }$ or $\theta_{\mathrm{D}}$ (e.g. Fulbright \& Reynolds 1990 considered only cases with $R_{\max }<2$ ), and we are considering remnants observed with a resolution greater than 10 beams per remnant diameter. Since in our models we follow the remnant evolution during the adiabatic phase, we also need to discard objects that are clearly in the radiative phase. Unfortunately, for most of the objects selected, there is no indication of their evolutionary stage in literature. Assuming that the remnant expands in a medium with particle number density $n_{\text {ism }} \lesssim 0.3 \mathrm{~cm}^{-3}$, the shock radius derived from the Sedov solution at time $t_{\text {tr }}$ (i.e. at the transition time from the adiabatic to the radiative phase; see Eq. (5)) is

$r_{\text {tr }}=19 E_{51}^{5 / 17} n_{\text {ism }}^{-7 / 17} \lesssim 35 \mathrm{pc}$, 

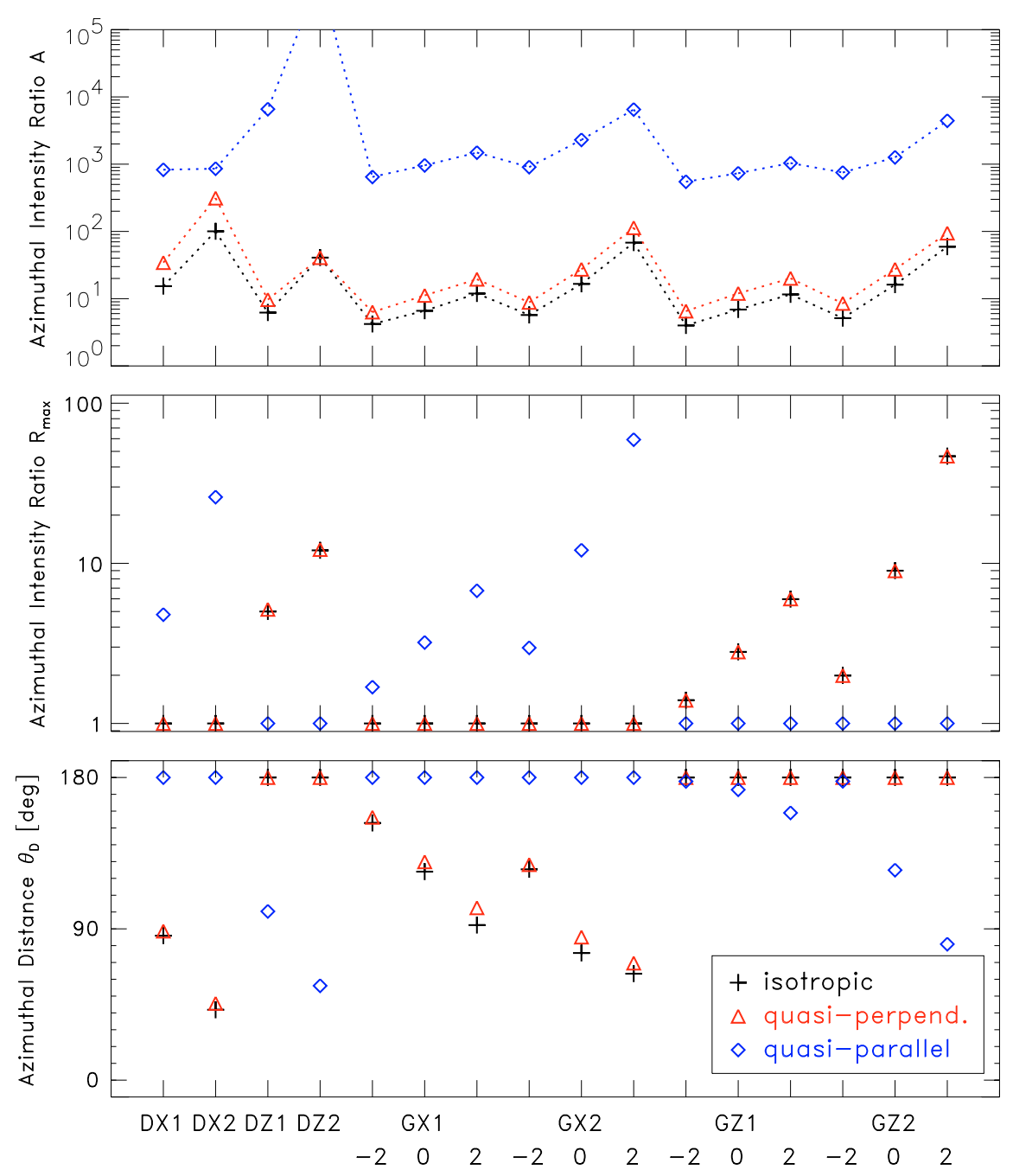

Fig. 8. Azimuthal intensity ratio $A$ (i.e. the ratio of maximum to minimum intensity around the shell of emission - see text; upper panel), azimuthal intensity ratio $R_{\max }$ (i.e. the ratio of the maxima of intensity of the two limbs around the shell; middle panel), and azimuthal distance $\theta_{\mathrm{D}}$ (i.e. the distance in deg of the two maxima of intensity around the shell; lower panel) for all the cases examined, considering the LoS aligned with the $y$ axis and a spatial resolution of 25 beams per remnant diameter, $D_{\mathrm{SNR}}$. Black crosses: isotropic; red triangles: quasiperpendicular; blue diamonds: quasi-parallel. where we have assumed that $E_{51}=1.5$. Therefore, we only considered remnants with radius $r_{\mathrm{snr}}<35 \mathrm{pc}$ (i.e. with size $<70 \mathrm{pc}$ ) that are, most likely, in the adiabatic phase. Our list does not pretend to be complete or representative of the class, and it is compiled to derive the observed values of the parameters $A, R_{\max }$, and $\theta_{\mathrm{D}}$ with the lowest uncertainties. For this reason, we have considered remnants for which a total intensity radio image in digital format is available. Actually, in most of the cases, we have used the $843 \mathrm{MHz}$ data of the MOST supernova remnant catalogue (Whiteoak \& Green 1996).

Our list is reported in Table 2. We have separated evolved and young SNRs. While the young SNRs listed in Table 2 have very reliable measurement of $A, R_{\max }$, and $\theta_{\mathrm{D}}$ and a good record of literature, making them very good candidates to test the diagnostic power of our model, we stress that the models we are considering in this paper are focused on evolved SNRs; we leave the discussion about young SNRs to a separate work. For each object in Table 2, we show the apparent size, the distance (from dedicated studies where possible, otherwise from the revised $\Sigma-D$ relation of Case \& Bhattacharya 1998; see their paper for caveats on usage of the $\Sigma-D$ relation to derive SNR distance), the real size, the resolution of the observation, and the parameters $A$, $R_{\max }$, and $\theta_{\mathrm{D}}$ we have introduced here.

Table 2 shows that most of the 11 remnants have $A \leq$ 10, i.e. values consistent with those derived in Fig. 8 for the three alternatives for the particle injection (recall that the values shown in the figure have to be considered as upper limits). Four remnants show high values of $A(10<A<100)$ that are difficult to explain in terms of the isotropic or the quasi-perpendicular injection models with $b<0$ unless the remnant expands through a non-uniform ambient magnetic field (see models DX2, and DZ2 in Fig. 8). In light of these considerations, we cannot exclude a priori any of the three alternative models for the particle injection.

Four of the 11 objects in Table 2 show values of $R_{\max } \geq 2$, pointing out that, in these objects, the bipolar morphology is asymmetric with the two radio limbs differing significantly in intensity. An example of this kind of remnants is G338.1+0.4 (see panel $\mathrm{C}$ in Fig. 6). In light of our results, its morphology can be explained if a gradient of ambient density or of ambient magnetic field strength is either perpendicular to the average ambient magnetic field, $\langle\boldsymbol{B}\rangle$, in the isotropic and quasi-perpendicular cases or parallel to $\langle\boldsymbol{B}\rangle$ in the quasi-parallel case. It is worth noting that revealing such a gradient from the observations may be a powerful diagnostic to discriminate among the alternative particle injection models, producing real advances in the understanding of the nonthermal physics of strong shock waves.

An extreme example of a monopolar remnant with a bright radio limb is G327.4+1.0 whose value of $R_{\max }$ is larger than 10 . Figure 8 shows that high values of $R_{\max }$ can easily be explained as being due to non-uniform ambient magnetic field strength or to non-uniform ambient density if $b>0$. We suggest that the morphology of G327.4+1.0 may give some hints on the value of $b$ (and, therefore, on the dependence of the injection efficiency 
Table 2. List of barrel-shaped SNR shells for which a measurement of $A, R_{\max }$, and $\theta_{\mathrm{D}}$ is presented for comparison with our models.

\begin{tabular}{lccccccccc}
\hline \hline Remnant $^{a}$ & $\begin{array}{c}\text { Flux } \\
\text { Jy }\end{array}$ & $\begin{array}{c}\text { size } \\
\text { arcmin }\end{array}$ & $\begin{array}{c}d \\
\mathrm{kpc}\end{array}$ & $\begin{array}{c}\text { size } \\
\mathrm{pc}\end{array}$ & $\begin{array}{c}\text { Res. } \\
\text { beams/ } D_{\text {SNR }}\end{array}$ & $A$ & $R_{\max }$ & $\begin{array}{c}\theta_{\mathrm{D}} \\
\mathrm{deg}\end{array}$ & Ref./notes \\
\hline G296.5+10.0 & 48 & $90 \times 65$ & 2.1 & $55 \times 40$ & 108 & $>11$ & 1.2 & 85 & 1 \\
G299.6-0.5 & 1.1 & $13 \times 13$ & 18.1 & 68 & 18 & 6 & 2 & 160 & 2 \\
G304.6+0.1 & 18 & $8 \times 8$ & 7.9 & 18 & 11 & 20 & 1.5 & 120 & 3 \\
G327.4+1.0 & 2.1 & $14 \times 13$ & 13.9 & 56 & 19 & $>10$ & $>10$ & ND & 2,4 \\
G332.0+0.2 & 8.9 & $12 \times 12$ & $<20$ & $<70$ & 17 & 5 & 1 & 145 & 2,7 \\
G338.1+0.4 & 3.8 & $16 \times 14$ & 9.9 & $46 \times 40$ & 21 & 3 & 2 & $>120$ & 2 \\
G341.9-0.3 & 2.7 & $7 \times 7$ & 14.0 & 28 & 10 & 8 & 3 & 170 & 2 \\
G346.6-0.2 & 8.7 & $11 \times 10$ & 8.2 & $26 \times 23$ & 15 & 2 & 1.1 & 110 & 2,7 \\
G351.7+0.8 & 11 & $18 \times 14$ & 6.7 & $35 \times 27$ & 22 & 2 & 1.6 & 130 & 2 \\
\hline & & & 70 & Young remnants & & & & \\
\hline G327.6+14.6 & 19 & $30 \times 30$ & 2.2 & $19 \times 19$ & 42 & 22 & 1 & 127 & 5 \\
G332.4-0.4 & 34 & $11 \times 10$ & 3.1 & $10 \times 9$ & 15 & 7 & 1.6 & 98 & 6 \\
\hline
\end{tabular}

References and notes. - (1) A.k.a. PKS 1209-51/52. Age: 3-20 kyr, Roger et al. (1988). Distance from Giacani et al. (2000). (2) Distance derived by Case \& Bhattacharya (1998) using a revised $\Sigma-D$ relation. (3) Distance from Caswell et al. (1975). (4) This shell has only one limb ("monopolar" according to the definition of Fulbright $\&$ Reynolds 1990). $A$ and $R_{\max }$ are lower limits and no $\theta_{\mathrm{D}}$ is derived. (5) A.k.a. SN1006. Distance from Winkler et al. (2003). (6) A.k.a. RCW103. Distance from Reynoso et al. (2004). (7) Two maxima have been found in one lobe. $\theta_{\mathrm{D}}$ is the average of the two.

${ }^{a}$ All the data are from the MOST supernova remnant catalogue (Whiteoak \& Green 1996), except where noted.

${ }^{b}$ Spatial resolution of the observation in beams per remnant diameter.

on the shock velocity) if the observations show that the asymmetry is due to a non-uniform ambient medium through which the remnant expands.

In Table 2, six of the 11 remnants (including the two young remnants SN1006 and RCW103) have values of $\theta_{\mathrm{D}}<140^{\circ}$, pointing out that, in these objects, the two bright radio limbs appear slanted and converging on one side. An example of this class of objects is G296.5+10.0 (a.k.a PKS 1209-51/52) shown in panel $\mathrm{F}$ in Fig. 6. In this case, the value of $\theta_{\mathrm{D}} \sim 85^{\circ}$ derived from the observations may be easily explained as being due to a gradient of magnetic field strength either parallel to $\langle\boldsymbol{B}\rangle$ in the isotropic and quasi-perpendicular cases or perpendicular to $\langle\boldsymbol{B}\rangle$ in the quasi-parallel case. Models with a gradient of ambient density cannot explain the low values of $\theta_{\mathrm{D}}$ found for G296.5+10.0 unless the gradients are strong (the density should change by a factor 60 over $60 \mathrm{pc}$ ) and the dependence of the injection efficiency on the shock velocity gives ${ }^{5} b \geq 2$.

\section{Conclusions}

Our findings have significant implications on the diagnostics and lead to several useful conclusions:

1. The three different particle injection models (namely, quasi-parallel, quasi-perpendicular, and isotropic dependence of injection efficiency from shock obliquity) can produce considerably different images (see Fig. 4). The isotropic and quasi-perpendicular cases lead to radio images similar to those observed. The parallel-case may produce radio images unlike any observed SNR (center-brightened or with a double-peak structure not describable as a shell). This is in agreement with the findings of Fulbright \& Reynolds (1990).

2. In models with gradients of the ambient density, the dependence of the injection efficiency on the shock velocity

\footnotetext{
${ }^{5}$ Large positive values of $b$ do not necessarily mean an increasing fraction of shock energy going into relativistic particles as the shock slows down because decelerating shock accelerates particles to smaller $E_{\max }$, namely the maximum energy at which the electrons are accelerated.
}

(through the parameter $b$ defined in Sect. 2.2) affects the degree of asymmetry of the radio images: the asymmetry increases with increasing value of $b$.

3. Small variations of the ambient magnetic field lead to significant asymmetries in the morphology of BSNRs (see Figs. 6 and 7). Therefore, we conclude that the close similarity of the radio brightness of the opposed limbs of a BSNR (i.e. $R_{\max } \approx 1$ and $\theta_{\mathrm{D}} \approx 180^{\circ}$ ) is evidence of uniform ambient $\boldsymbol{B}$ field where the remnant expands.

4. Variations of the ambient density lead to asymmetries of the remnant with extent comparable to that caused by nonuniform ambient magnetic field if $b=2$.

5. Strongly asymmetric BSNRs (i.e. $R_{\max } \gg 1$ or $\theta_{\mathrm{D}} \ll 180^{\circ}$ ) imply either moderate variations of $\boldsymbol{B}$ or strong (moderate) variations of the ISM density if $b<2(b \geq 2)$ as in the case, for instance, of interaction with a giant molecular cloud.

6. BSNRs with different intensities of the emission of the radio arcs (i.e. $R_{\max }>1$ ) can be produced by models with a gradient of density or of magnetic field strength perpendicular to the arc (upper panels in Fig. 6 and lower panels in Fig. 7), and the brightest arc is in the region of higher plasma density or higher magnetic field strength.

7. Remnants with two slanting similar arcs (i.e. $\theta_{\mathrm{D}}<180^{\circ}$ ) can be produced by models with a gradient of density or of magnetic field strength running centered between the two arcs (lower panels in Fig. 6 and upper panels in Fig. 7); the region of convergence is where either the plasma density or the magnetic field strength is higher.

8. In all the cases examined, the symmetry axis of the remnant is always aligned with the gradient of density or of magnetic field.

We found that the degree of ordering of the magnetic field downstream of the shock does not significantly affect the asymmetries induced by gradients either of ambient plasma density or of ambient magnetic field strength; thus our conclusions, derived in the case of disordered magnetic field, do not change in the case of ordered magnetic fields.

We defined useful model parameters to quantify the degree of asymmetry of the remnants. These parameters may provide 
a powerful diagnostic in the comparison between models and observations, as we have shown in a few cases drawn from a randomly selected sample of BSNRs presented in Table 2. For instance, if the density of the external medium is known by other means (e.g. thermal X-rays, H I and CO maps, etc.), BSNRs can be very useful in investigating the variation of the efficiency of electron injection with the angle between the shock normal and the ambient magnetic field or in investigating the dependence of the injection efficiency from the shock velocity. Alternatively, BSNRs can be used as probes to trace the local configuration of the galactic magnetic field if the dependence of the injection efficiency from the obliquity is known.

It is worth emphasizing that our models follow the evolution of the remnant during the adiabatic phase and, therefore, their applicability is limited to this evolutionary stage. In the radiative phase, the high degree of compression suggested by radiative shocks leads to increase in the radio brightness due to compression of ambient magnetic field and electrons. Since our model neglects the radiative cooling, it is limited to relatively small compression ratios and, therefore, it is not able to simulate this mechanism of limb brightening.

It will be interesting to expand the present study, considering the detailed comparison of model results with observations. This may lead to a major advance in the study of interactions between the magnetized ISM and the whole galactic SNR population (not only BSNRs), since the mechanisms at work in the BSNRs are also valid for SNRs of more complex morphology.

Acknowledgements. We thank the referee for constructive and helpful criticism. The software used in this work was in part developed by the DOE-supported ASC/Alliance Center for Astrophysical Thermonuclear Flashes at the University of Chicago. The simulations were executed at CINECA (Bologna, Italy) in the framework of the INAF-CINECA agreement. This work was supported by Ministero dell'Istruzione, dell'Università e della Ricerca, by Istituto Nazionale di Astrofisica, and by Agenzia Spaziale Italiana (ASI/INAF I/023/05/0).

\section{Appendix A: Derivation of Eq. (8)}

We follow Reynolds (1998) in the description of the evolution of electron distribution. His approach is extended here to the possibility to deal with non-uniform ISM (cf. Petruk 2006). Fluid element $a \equiv R\left(t_{\mathrm{i}}\right)$ was shocked at time $t_{\mathrm{i}}$, where $R$ is the radius of the shock, and $a$ is the Lagrangian coordinate. At that time the electron distribution on the shock was

$N\left(E_{\mathrm{i}}, a, t_{\mathrm{i}}\right)=K_{\mathrm{s}}\left(a, t_{\mathrm{i}}\right) E_{\mathrm{i}}^{-\zeta}$,

where $E_{\mathrm{i}}$ is the electron energy at time $t_{\mathrm{i}}, K_{\mathrm{s}}$ is the normalization of the electron distribution immediately after the shock (in the following, index "s" refers to the immediately post-shock values), and $\zeta$ is the power law index. Since we are interested in radio emission, we have to account for energy losses of electrons due only to the adiabatic expansion (Reynolds 1998):

$\frac{\mathrm{d} E}{\mathrm{~d} t}=\frac{E}{3 \rho} \frac{\mathrm{d} \rho}{\mathrm{d} t}$,

where $\rho$ is the mass density, so the energy varies as

$E=E_{\mathrm{i}}\left(\frac{\rho(a, t)}{\rho_{\mathrm{s}}\left(a, t_{\mathrm{i}}\right)}\right)^{1 / 3}$.

The conservation law for the number of particles per unit volume per unit energy interval

$N(E, a, t)=N\left(E_{\mathrm{i}}, a, t_{\mathrm{i}}\right) \frac{a^{2} \mathrm{~d} a \mathrm{~d} E_{\mathrm{i}}}{\sigma r^{2} \mathrm{~d} r \mathrm{~d} E}$, where $\sigma$ is the shock compression ratio and $r$ is the Eulerian coordinate, together with the continuity equation $\rho_{\mathrm{o}}(a) a^{2} \mathrm{~d} a=$ $\rho(a, t) r^{2} \mathrm{~d} r$ (index "o" refers to the pre-shock values) and the derivative

$\frac{\mathrm{d} E_{\mathrm{i}}}{\mathrm{d} E}=\left(\frac{\rho(a, t)}{\rho_{\mathrm{s}}\left(a, t_{\mathrm{i}}\right)}\right)^{-1 / 3}$,

implies that downstream

$N(E, a, t)=K_{\mathrm{s}}\left(a, t_{\mathrm{i}}\right) E^{-\zeta}\left(\frac{\rho(a, t)}{\rho_{\mathrm{s}}\left(a, t_{\mathrm{i}}\right)}\right)^{(\zeta+2) / 3}$.

If $K_{\mathrm{s}} \propto \rho_{\mathrm{s}} V_{\mathrm{sh}}(t)^{-b}$, where $V_{\mathrm{sh}}(t)$ is the shock velocity and $\rho_{\mathrm{s}}$ is the immediately post-shock value of density, then

$K_{\mathrm{s}}\left(a, t_{\mathrm{i}}\right)=K_{\mathrm{s}}(R, t)\left(\frac{\rho_{\mathrm{o}}(a)}{\rho_{\mathrm{o}}(R)}\right)\left(\frac{V_{\mathrm{sh}}(t)}{V_{\mathrm{sh}}\left(t_{\mathrm{i}}\right)}\right)^{b}$.

Therefore, the distribution of relativistic electrons follows

$\frac{K(a, t)}{K_{\mathrm{s}}(R, t)}=\frac{N(E, a, t)}{N(E, R, t)}=\left(\frac{\rho_{\mathrm{o}}(a)}{\rho_{\mathrm{o}}(R)}\right)\left(\frac{V_{\mathrm{sh}}(t)}{V_{\mathrm{sh}}\left(t_{\mathrm{i}}\right)}\right)^{b}\left(\frac{\rho(a, t)}{\rho_{\mathrm{s}}\left(a, t_{\mathrm{i}}\right)}\right)^{(\zeta+2) / 3}$.

Now we can substitute Eq. (A.8) with the ratio of the shock velocities which comes from the expression (Hnatyk \& Petruk 1999)

$\frac{P(a, t)}{P_{\mathrm{s}}(R, t)}=\left(\frac{\rho_{\mathrm{o}}(a)}{\rho_{\mathrm{o}}(R)}\right)^{-2 / 3}\left(\frac{V_{\mathrm{sh}}\left(t_{\mathrm{i}}\right)}{V_{\mathrm{sh}}(t)}\right)^{2}\left(\frac{\rho(a, t)}{\rho_{\mathrm{s}}(R, t)}\right)^{5 / 3}$.

Thus, finally

$$
\begin{aligned}
\frac{K(a, t)}{K_{\mathrm{s}}(R, t)}= & \left(\frac{P(a, t)}{P_{\mathrm{s}}(R, t)}\right)^{-b / 2} \\
& \times\left(\frac{\rho_{\mathrm{o}}(a)}{\rho_{\mathrm{o}}(R)}\right)^{-(b+\zeta-1) / 3}\left(\frac{\rho(a, t)}{\rho_{\mathrm{s}}(R, t)}\right)^{5 b / 6+(\zeta+2) / 3} .
\end{aligned}
$$

This formula may easily be used to calculate the profile of $K(a)$ for known $P(a)$ and $\rho(a)$ in the case of the radial flow of fluid. In the case when mixing is allowed, the position $\boldsymbol{R}$ should correspond to the same part of the shock which was at $\boldsymbol{a}$ at time $t_{\mathrm{i}}$.

\section{References}

Balsara, D., Benjamin, R. A., \& Cox, D. P. 2001, ApJ, 563, 800 Bell, A. R., \& Lucek, S. G. 2001, MNRAS, 321, 433

Blondin, J. M., Wright, E. B., Borkowski, K. J., \& Reynolds, S. P. 1998, ApJ, 500,342

Case, G. L., \& Bhattacharya, D. 1998, ApJ, 504, 761

Caswell, J. L., Murray, J. D., Roger, R. S., Cole, D. J., \& Cooke, D. J. 1975, A\&A, 45, 239

Dickel, J. R., van Breugel, W. J. M., \& Strom, R. G. 1991, AJ, 101, 2151

Dohm-Palmer, R. C., \& Jones, T. W. 1996, ApJ, 471, 279

Ellison, D. C., Baring, M. G., \& Jones, F. C. 1995, ApJ, 453, 873

Fryxell, B., Olson, K., Ricker, P., et al. 2000, ApJS, 131, 273

Fulbright, M. S., \& Reynolds, S. P. 1990, ApJ, 357, 591

Gaensler, B. M. 1998, ApJ, 493, 781

Giacani, E. B., Dubner, G. M., Green, A. J., Goss, W. M., \& Gaensler, B. M. 2000, AJ, 119, 281

Ginzburg, V. L., \& Syrovatskii, S. I. 1965, ARA\&A, 3, 297

Hnatyk, B., \& Petruk, O. 1999, A\&A, 344, 295

Jokipii, J. R. 1987, ApJ, 313, 842

Jun, B.-I., \& Norman, M. L. 1996, ApJ, 472, 245

Kane, J., Drake, R. P., \& Remington, B. A. 1999, ApJ, 511, 335

Kesteven, M. J., \& Caswell, J. L. 1987, A\&A, 183, 118 
Leckband, J. A., Spangler, S. R., \& Cairns, I. H. 1989, ApJ, 338, 963 Löhner, R. 1987, Comp. Meth. Appl. Mech. Eng., 61, 323 Lucek, S. G., \& Bell, A. R. 2000, MNRAS, 314, 65

Mac Low, M.-M., \& Klessen, R. S. 2004, Rev. Mod. Phys., 76, 125

MacNeice, P., Olson, K. M., Mobarry, C., de Fainchtein, R., \& Packer, C. 2000 Comp. Phys. Comm., 126, 330

Mineshige, S., \& Shibata, K. 1990, ApJ, 355, L47

Ostrowski, M. 1988, MNRAS, 233, 257

Petruk, O. 2005, J. Phys. Studies, 9, 364

Petruk, O. 2006, A\&A, 460, 375

Petruk, O., \& Bandiera, R. 2006, J. Phys. Studies, 10, 66

Reynolds, S. P. 1998, ApJ, 493, 375
Reynolds, P. S., \& Fulbright, S. M. 1990, in International Cosmic Ray Conference, 72

Reynolds, S. P., \& Gilmore, D. M. 1993, AJ, 106, 272

Reynoso, E. M., Green, A. J., Johnston, S., et al. 2004, Public. Astron. Soc. Aust., 21,82

Roger, R. S., Milne, D. K., Kesteven, M. J., Wellington, K. J., \& Haynes, R. F. 1988, ApJ, 332, 940

Rothenflug, R., Ballet, J., Dubner, G., et al. 2004, A\&A, 425, 121

Truelove, J. K., \& McKee, C. F. 1999, ApJS, 120, 299

Whiteoak, J. B. Z., \& Green, A. J. 1996, A\&AS, 118, 329

Winkler, P. F., Gupta, G., \& Long, K. S. 2003, ApJ, 585, 324 